\title{
Obsessive-compulsive disorder as a visual processing impairment
}

\author{
Óscar F. Gonçalves ${ }^{\mathrm{a}, *}$, Tiago Reis Marques ${ }^{\mathrm{b}, \mathrm{c}}$, Nicolás F. Lori ${ }^{\mathrm{d}}$, Adriana Sampaio ${ }^{\mathrm{a}}$, Miguel Castelo Branco ${ }^{\mathrm{d}}$ \\ ${ }^{a}$ Neuropsychophysiology Laboratory, CIPSI, School of Psychology, University of Minho, 4700 Braga, Portugal \\ ${ }^{\mathrm{b}}$ Institute of Psychiatry, University of London, UK \\ ${ }^{\mathrm{c}}$ University Hospital of Coimbra, Portugal \\ ${ }^{\mathrm{d}}$ Visual Neuroscience Laboratory, IBILI, Faculty of Medicine, University of Coimbra, Portugal
}

\section{A R T I C L E I N F O}

\section{Article history:}

Received 22 July 2009

Accepted 26 July 2009

\begin{abstract}
S U M M A R Y
OCD has been hypothesized to involve the failures in both cognitive and behavioral inhibitory processes. There is evidence that the hyperactivation of cortical-subcortical pathways may be involved in the failure of these inhibitory systems associated with OCD. Despite this consensus on the role of frontal-subcortical pathways in OCD, recent studies have been showing that brain regions other than the frontal-subcortical loops may be needed to understand the different cognitive and emotional deficits in OCD. Some studies have been finding evidence for decreased metabolic activity in areas such as left inferior parietal and parieto-occipital junction suggesting the possible existence of visual processing deficits. While there has been inconsistent data regarding visual processing in OCD, recent studies have been claiming that these patients have abnormal patterns of visual processing social rich stimuli, particularly emotional arousing stimuli. Thus, in this article, we hypothesize that the fronto-subcortical activation consistently found in OCD may be due to a deactivation of occipital/parietal regions associated with visual-perceptual processing of incoming social rich stimuli. Additionally, this dissociation may be more evident as the emotional intensity of the social stimulus increases.
\end{abstract}

(ㄷ) 2009 Elsevier Ltd. All rights reserved.

\section{Introduction}

OCD is an anxiety disorder characterized by the presence of intrusive negative thoughts, images, or ideas, involuntarily entering consciousness (obsessions) which are often accompanied by repetitive, stereotyped or ritualized behaviors (compulsions).

OCD is probably one of the most disabling psychological disorders with a consistent cross cultural lifetime prevalence of about $2.5 \%[1,2]$.

In terms of neurocognitive processes, OCD has been hypothesized to involve the failures in two main inhibitory processes: (a) cognitive inhibitory process (responsible for obsessive symptoms); and (b) behavioral inhibitory process (responsible for compulsive symptoms) [3].

The failures of these two inhibitory systems may mediate not only OCD cognitive and behavioral symptoms but also most of the neuropsychological deficits found in this disorder in terms of attention, memory, planning and decision making [3-5].

Several distinct pathophysiological mechanisms have been associated with OCD at the neuroimmunological [6], neurochemical [7], and neuroanatomic levels [8]. A variety of neuroanatomic models have been proposed to explain the pathogenesis of OCD

\footnotetext{
* Corresponding author. Tel.: +351 253604261; fax: +351 253604221

E-mail address: goncalves@iep.uminho.pt (Ó.F. Gonçalves).
}

[9-11] but they tend to agree on the role of cortico-basal ganglia-thalamus interaction.

Contemporary models of OCD pathogenesis acknowledge that two cortical-subcortical pathways may be involved in the failure of the inhibitory systems: (a) the frontostriatal loop (dorsolateral-caudate-striatum-thalamus) responsible for failures of behavioral inhibition; (b) the orbitofrontal loop (orbitofrontal, medial prefrontal and cingulate) responsible for failures with cognitive inhibitory processes $[12,13]$.

In fact, structural MRI studies confirm the existence of volumetric changes in these cortical-subcortical networks. A recent meta-analysis found evidence of significant volumetric reductions on the left anterior cingulated and bilateral orbitofrontal cortex, contrasting with an increase in left and right thalamic volumes [14].

These structural changes may be the consequence of the hyperactivation of frontal-subcortical circuits often found in neurofunctional studies. For example, a meta-analysis of the studies using PET found significant differences in radiotracer uptake between OCD and controls suggesting an increase in activity in the left orbital gyrus and the left and right head of the caudate nucleus [8]. Not differently, a recent review by Rotge et al. [15] on functional neuroimaging of provocation of OCD symptoms corroborates the consensus on the activation of the orbitofrontal and anterior cingulated loops. 
Despite this consensus on the role of frontal-subcortical activation in $\mathrm{OCD}$, recent studies have been showing that brain regions other than the frontal-subcortical loops may be needed to understand the different cognitive and emotional deficits in OCD. In fact, a recent quantitative voxel-level meta-analysis of fMRI case-control OCD studies by Menzies et al. [16] brings evidence for the need to consider the role of other posterior brain regions in the pathogenesis of OCD. For example, Nordahl et al. [17] found a below-normal glucose metabolism in the occipital-parietal area contrasting with increased glucose metabolism in the left orbital frontal, right sensorimotor, and bilateral prefrontal and anterior cingulate regions. More recently, Kwon et al. [18] found a decreased metabolic activity in left inferior parietal and parieto-occipital junction contrasting with orbitofrontal hyper-metabolism in OCD patients.

In another study a whole brain voxel-based morphometry conducted by van den Heuvel [19] showed that multiple symptomatic dimensions of OCD may be associated with specific structural substrates both in terms of grey and white matter. Even though overall volume reductions were found for both white matter and grey matter in the cortical-subcortical networks, specific patterns of structural changes were found to be associated with different symptomatic configurations such as: symmetry/ordering (e.g., negatively correlated with right motor cortex grey matter; left insula and left parietal cortex); contamination/washing (e.g., negatively correlated with bilateral caudate nucleus and white matter volume in right parietal region); harm/checking (e.g., negatively correlated with bilateral temporal lobes white matter).

Not differently, diffusion tensor imaging (DTI) studies analyzing connectivity patterns in patients with OCD showed lower fractional anisotropy (FA) values in anterior and posterior cingulate gyrus, as well as bilaterally in parietal regions and in occipital lobe $[20,21]$. The lower FA values in the parietal region correlated significantly with higher Yale-Brown obsessive-compulsive scale scores [21]. Interestingly, these frontal and parietal white matter abnormalities were also exhibited by first relatives of patients with OCD [22].

Consistent with this, spectroscopy studies bring additional support to the involvement of parietal white matter abnormality in OCD in finding that choline-containing compounds and creatine/ phosphocreatine were found to be significantly higher in the parietal cortex of OCD patients [23].

Altogether, there is initial evidence that areas involved in visual processing may be impaired in OCD. However, studies of visual processing results in OCD have provided inconsistent data with some authors claiming the existence of visual impairments [24] while others claim that visual processing deficits may be overestimated [25]. We believe that these inconsistencies are due to the abstract/conceptual nature of most of the stimuli used in the situation. We believe that moving visual stimuli closer to an ecological and emotional context will show evidence of visual processing impairment.

A recent study by Jung et al. [26] brings initial evidence for this hypothesis by showing that in a more social stimuli situation such as biological motion, OCD patients show an increased activation of the superior, middle, inferior temporal and fusiform gyrus as well as cerebellum and a hypoactivation in the postcentral region.

The need to study visual processing deficit in emotional contexts was recently demonstrated by Moritz et al. [27] finding that, in OCD patients, visual attention was affected both at early and late stages of processing when OCD relevant emotional stimuli are used.

Finally, in a clinical study, Kang et al. [28] found that improvement after SSRI psychopharmacological treatment was associated with improvement in performance of Rey-Osterrieth complex figure test. Additionally, this improvement was associated with decreased metabolism in frontal-subcortical and increased metab- olism in parietal-occipital regions (e.g., lateral right postcentral gyrus; posterior region of the superior parietal lobe; medial portion of the superior occipital gyrus). Not differently, Nabeyama et al. [29] found that after effective treatment with behavior therapy, OCD patients showed, in a Stroop task, increased activation of the posterior regions such as parietal cortex and a decrease in the activation of frontal regions such as orbitofrontal cortex and middle frontal gyrus.

\section{The hypothesis}

In sum, based on current findings, one may hypothesize that the fronto-subcortical activation consistently found in OCD may be due to a deactivation of occipital/parietal regions associated with visual-perceptual processing of incoming social rich stimuli.

Additionally, this dissociation may be more evident as the emotional intensity of the social stimulus increases. In fact studies have shown that the presentation of emotional pictures (e.g., IAPS) is responsible for increased activation of the visual cortex in healthy individuals when compared with non-emotional pictures [30] and that the level of picture affective arousal correlates positively with functional activity [30,31]. Additionally, the extent and strength of visual cortex activation was found to be greater for images with "erotic" and "mutilation" content [31,32]. These effects were particularly evident in visual secondary association areas BA $[18,19]$ and fusyform gyrus.

Contrary to what is seen in normal healthy controls, OCD patients have a reversal pattern, with an increased activation in the frontal areas for the presentation of eliciting emotional arousal associated with their obsessions/compulsions [33].

Altogether, we believe there is initial evidence to advance the following hypothesis concerning the pathophysiology of OCD:

1. The fronto-subcortical activation consistently found in OCD may be due to a correlative of a deactivation of occipital/parietal regions associated with visual-perceptual processing.

2. The impairment of visual processing deficits may be restricted to social rich stimuli and as such be more evident on visual cortical association areas.

3. The level of visual processing deficit may be correlative on the level of emotional arousal of the social stimuli.

\section{Implications and further studies}

If future research validates these hypotheses, important implications may be derived for the development of new therapeutic approaches. For instances, cognitive therapy methods should be aimed to increase the efficiency of visual processing for emotional laden stimuli rather than using distractive or thought-stopping techniques. In psychopharmacological treatments, all medications that might interfere with visual processing abilities must be avoided. Finally, more recent methods, such as transcranial magnetic stimulation, can be used to activate visual processing areas and deactivate frontal-subcortical circuits while the patient is confronted with emotional triggers.

\section{Conflicts of interest statement}

There are not any financial, relationship and organizational conflict of interests that may bias any of the authors in the establishment of the hypotheses discussed in this article.

\section{References}

[1] Stein DJ. Obsessive-compulsive disorder. Lancet 2002;360:397-405. 
[2] Weissman MM, Bland RC, Canino GJ, et al. The cross national epidemiology of obsessive compulsive disorder. J Clin Psychiatry 1994;55(Suppl.):5-10.

[3] Chamberlain SR, Blackwell AD, Fineberg NA, Robbins TW, Sahakian BJ. The neuropsychology of obsessive compulsive disorder: the importance of failures in cognitive and behavioural inhibition as candidate endophenotypic markers. Neurosci Biobehav Rev 2005;29:399-419.

[4] Rao NP, Reddy YC, Kumar KJ, Kandavel T, Chandrashekar CR. Are neuropsychological deficits trait markers in OCD? Prog Neuropsychopharmacol Biol Psychiatry 2008;32:1574-9.

[5] Shin MS, Choi H, Kim H, Hwang JW, Kim BN, Cho SC. A study of neuropsychological deficit in children with obsessive-compulsive disorder. Eur Psychiatry 2008;23:512-20.

[6] da Rocha FF, Correa H, Teixeira AL. Obsessive-compulsive disorder and immunology: a review. Prog Neuropsychopharmacol Biol Psychiatry 2008;32:1139-46

[7] Hesse S, Müller U, Lincke T, et al. Serotonin and dopamine transporter imaging in patients with obsessive-compulsive disorder. Psychiatry Res: Neuroimaging 2005; $140: 63-7$.

[8] Whiteside SP, Port JD, Abramowitz JS. A meta-analysis of functional neuroimaging in obsessive-compulsive disorder. Psychiatry Res: Neuroimaging 2004;132:69-79.

[9] Baxter LR. Functional imaging of brain systems mediating obsessivecompulsive disorder: clinical studies. In: Charney DS, Nestler EJ, Bunney BS, editors. Neurobiology of mental illness. New York: Oxford University Press; 1999. p. 534-47.

[10] Modell JG, Mountz JM, Curtis GC, Greden JF. Neurophysiologic dysfunction in basal ganglia/limbic striatal and thalamocortical circuits as a pathogenetic mechanism of obsessive-compulsive disorder. J Neuropsychiatry Clin Neurosci 1989;1:27-36.

[11] Schwartz JM. A role of volition and attention in the generation of new brain circuitry. Toward a neurobiology of mental force. J Conscious Stud 1999;6:115-42.

[12] Aouizerate B, Guehl D, Cuny E, et al. Pathophysiology of obsessive-compulsive disorder: a necessary link between phenomenology, neuropsychology, imagery and physiology. Prog Neurobiol 2004;72:195-221.

[13] Friedlander L, Desrocher M. Neuroimaging studies of obsessive-compulsive disorder in adults and children. Clin Psychol Rev 2006;26:32-49.

[14] Rotge JY, Guehl D, Dilharreguy B, et al. Meta-analysis of brain volume changes in obsessive-compulsive disorder. Biol Psychiatry 2009;65:75-83.

[15] Rotge JY, Guehl D, Dilharreguy B, et al. Provocation of obsessive-compulsive symptoms: a quantitative voxel-based meta-analysis of functional neuroimaging studies. J Psychiatry Neurosci 2008;33:405-12.

[16] Menzies L, Chamberlain SR, Laird AR, Thelen SM, Sahakian BJ, Bullmore ET. Integrating evidence from neuroimaging and neuropsychological studies of obsessive-compulsive disorder: the orbitofronto-striatal model revisited. Neurosci Biobehav Rev 2008;32:525-49.

[17] Nordahl TE, Benkelfat C, Semple WE, Gross M, King AC, Cohen RM. Cerebral glucose metabolic rates in obsessive compulsive disorder. Neuropsychopharmacology 1989;2:23-8.
[18] Kwon JS, Kim JJ, Lee DW, et al. Neural correlates of clinical symptoms and cognitive dysfunctions in obsessive-compulsive disorder. Psychiatry Res 2003;122:37-47.

[19] van den Heuvel OA, Remijnse PL, Mataix-Cols D, et al. The major symptom dimensions of obsessive-compulsive disorder are mediated by partially distinct neural systems. Brain 2009;132:853-68.

[20] Cannistraro PA, Makris N, Howard JD, et al. A diffusion tensor imaging study of white matter in obsessive-compulsive disorder. Depress Anxiety 2007;24: 440-6.

[21] Szeszko PR, Ardekani BA, Ashtari M, et al. White matter abnormalities in obsessive-compulsive disorder: a diffusion tensor imaging study. Arch Gen Psychiatry 2005;62:782-90.

[22] Menzies L, Williams GB, Chamberlain SR, et al. White matter abnormalities in patients with obsessive-compulsive disorder and their first-degree relatives. Am J Psychiatry 2008;165:1308-15.

[23] Kitamura H, Shioiri T, Kimura T, Ohkubo M, Nakada T, Someya T. Parietal white matter abnormalities in obsessive-compulsive disorder: a magnetic resonance spectroscopy study at 3-Tesla. Acta Psychiatr Scand 2006;114:101-8.

[24] Mataix-Cols D, Alonso P, Hernandez R, et al. Relation of neurological soft signs to nonverbal memory performance in obsessive-compulsive disorder. J Clin Exp Neuropsychol 2003;25:842-51.

[25] Moritz S, Kloss M, Jacobsen D, et al. Extent, profile and specificity of visuospatial impairment in obsessive-compulsive disorder (OCD). J Clin Exp Neuropsychol 2005;27:795-814.

[26] Jung WH, Gu BM, Kang DH, et al. BOLD response during visual perception of biological motion in obsessive-compulsive disorder: an fMRI study using the dynamic point-light animation paradigm. Eur Arch Psychiatry Clin Neurosci 2009;259:46-54.

[27] Moritz S, Von Muhlenen A, Randjbar S, Fricke S, Jelinek L. Evidence for an attentional bias for washing- and checking-relevant stimuli in obsessivecompulsive disorder. J Int Neuropsychol Soc 2009;15:365-71.

[28] Kang DH, Kwon JS, Kim JJ, et al. Brain glucose metabolic changes associated with neuropsychological improvements after 4 months of treatment in patients with obsessive-compulsive disorder. Acta Psychiatr Scand 2003;107:291-7

[29] Nabeyama M, Nakagawa A, Yoshiura T, et al. Functional MRI study of brain activation alterations in patients with obsessive-compulsive disorder after symptom improvement. Psychiatry Res 2008;163:236-47.

[30] Lang PJ, Bradley MM, Fitzsimmons JR, et al. Emotional arousal and activation of the visual cortex: an fMRI analysis. Psychophysiology 1998;35:199-210.

[31] Bradley MM, Sabatinelli D, Lang PJ, Fitzsimmons JR, King W, Desai P. Activation of the visual cortex in motivated attention. Behav Neurosci 2003;117:369-80.

[32] Sabatinelli D, Bradley MM, Fitzsimmons JR, Lang PJ. Parallel amygdala and inferotemporal activation reflect emotional intensity and fear relevance. Neuroimage 2005;24:1265-70.

[33] Mataix-Cols D, Wooderson S, Lawrence N, Brammer MJ, Speckens A, Phillips ML. Distinct neural correlates of washing, checking, and hoarding symptom dimensions in obsessive-compulsive disorder. Arch Gen Psychiatry 2004;61:564-76 